\title{
Estimation des paramètres génétiques de la résistance au parasitisme gastro-intestinal en élevage villageois de petits ruminants au Sénégal
}

\author{
V. Clément $1^{*}$ J. Bouix ${ }^{1}$ E. Tillard ${ }^{2}$ \\ A. Gueye ${ }^{3}$ R.L. Baker ${ }^{4}$
}

Mots-clés

O vin - Caprin - Méthode d'élevage Parasites - Maladie de l'appareil digestif - Paramètres génétiques Sénégal.

\begin{abstract}
Résumé
Un protocole d'étude de la résistance génétique aux strongyloses gastro-intestinales a été mis en place sur des races locales d'ovins et de caprins du Sénégal. Des prélèvements de sang et des coproscopies ont été réalisés pendant deux ans sur des animaux de différents troupeaux de la zone de Louga en climat sahélien et de la zone de Kolda en climat soudano-guinéen. L'identité des pères étant inconnue dans ce contexte d'élevage, les apparentements étudiés étaient essentiellement les relations mère-descendant. Les caractères analysés étaient la coproscopie (trois prélèvements par an) en nombre d'œufs par gramme de fèces $(0 \mathrm{pg})$ et l'hématocrite (deux prélèvements par an). Dans la région de Louga, le nombre d'individus étudiés (des deux espèces) était de 377 la première année et 606 la deuxième année. Dans la zone de Kolda, l'échantillon comprenait 671 animaux la première année, 725 la deuxième année. L'héritabilité et la répétabilité ont été estimées à l'aide d'un modèle « animal », avec comme effets fixés la combinaison village*saison*année, l'âge et le stade de lactation pour les mères, la combinaison village*saison*année, l'âge et le sexe pour les jeunes. La répétabilité moyenne intra-année était de 0,19 pour le caractère nombre d'O pg et 0,27 pour I'hématocrite. Elle était plus faible entre années : 0,12 pour le nombre d'O pg et 0,21 pour l'hématocrite. L'estimation de l'héritabilité a présenté des difficultés, à cause de la structure des données villageoises qui ne permettait pas d'estimer directement l'environnement maternel et les effets troupeaux, respectivement en raison de généalogies insuffisantes et de faibles effectifs par élevage. Les estimations de I'héritabilité variaient entre 0 et 0,33 pour le nombre d'O pg et entre 0 et 0,38 pour I'hématocrite selon I'échantillon analysé et le modèle utilisé. Les corrélations génétiques entre caractères n'ont pas pu être estimées. En revanche, les corrélations phénotypiques ont été estimées entre le nombre d'Opg et l'hématocrite, ainsi qu'entre chacune de ces deux variables et le poids des jeunes. Les contraintes liées à une étude génétique en milieu traditionnel ont été discutées.
\end{abstract}

\section{INTRODUCTION}

Les parasites gastro-intestinaux, présents au stade larvaire sur le pâturage, sont responsables chez les ruminants de taux de mortalité élevés et d'une baisse de la productivité (chute de poids, ralentissement de la croissance, réduction de la fertilité... ). Les traitements chimiques sont coûteux, néfastes pour l'environnement et, à plus long terme, sont à l'origine du développement de mécanismes de résistance chez les parasites $(14,21,27,33)$. L'élevage d'animaux

1. Inra-Saga, BP 27, 31326 Castanet-Tolosan Cedex, France. Tél : +330561285194; Fax : + 330561285353 ; E-mail : clement@germinal.toulouse.inra.fr

2. Cirad-Elevage, 97410 Saint-Pierre, La Réunion

3. Isra-Lnerv, BP 2057, Dakar Hann, Sénégal

4. Ilri, PO Box 30709, Nairobi, Kenya

* Auteur pour la correspondance génétiquement résistants pourrait être une bonne alternative aux traitements chimiques : l'identification et la sélection d'animaux, sur la base de critères de résistance à l'infestation, est un moyen efficace de réduire les pertes causées par les parasites gastro-intestinaux $(4,9,17,32)$. Par ailleurs, certaines races tropicales s'avèrent être plus résistantes que d'autres et il est vraisemblable que ce soit une résultante de la sélection naturelle (7). Les critères couramment utilisés pour étudier la résistance au parasitisme gastrointestinal sont de deux types. Ils permettent d'évaluer soit la résistance des hôtes face à l'installation et au développement des parasites, soit la capacité des animaux à lutter contre les conséquences d'une infestation. La première série de critères, nombre d'Opg et hématocrite, concerne la mesure du niveau d'infestation par les parasites, l'hématocrite traduisant le niveau d'anémie de l'hôte infesté par des parasites hématophages. La deuxième série de critères est relative aux variables de production de l'animal hôte 
soumis à une infestation, telles que le poids vif des jeunes d'où l'on déduit la production laitière des mères.

En climat tropical, la chaleur et l'humidité sont particulièrement favorables au développement des larves et le taux de prévalence est souvent proche de 100 p. 100 (12, 22). Au Sénégal notamment, le parasitisme gastro-intestinal est une pathologie majeure chez les ruminants, d'autant plus que l'élevage est en grande majorité de type extensif et que les animaux sont en contact direct avec les parasites sur le pâturage. Dans les élevages traditionnels, le pourcentage d'animaux parasités est élevé (de 27 à 88 p. 100 en Casamance en saison des pluies) (15) et les pertes de poids vif peuvent atteindre 10 à 50 p. 100 chez les ovins (31). L'amélioration génétique des animaux pourrait être à long terme un moyen efficace de lutter contre le parasitisme gastro-intestinal dans les pays tropicaux.

L'objectif de ce travail était double. D'une part, il s'agissait d'estimer l'héritabilité et la répétabilité des caractères de résistance (nombre d'Opg et hématocrite) en infestation naturelle chez des ovins et des caprins dans un système d'élevage villageois au Sénégal. D'autre part, il s'agissait de discuter des problèmes méthodologiques rencontrés et des conditions de réussite d'une étude génétique dans un contexte d'élevage traditionnel tel que celui-ci. Des données de parasitologie ont été recueillies dans des troupeaux villageois situés en zones sahélienne et soudano-guinéenne par le projet de recherche conjoint Cirad-emvt/Isra (programme Ppr : Pathologie et productivité des petits ruminants), dans le cadre de l'Action thématique programmée : Strongyloses gastro-intestinales des petits ruminants en milieu tropical, résistance génétique et milieu d'infestation.

\section{MATERIEL ET METHODES}

\section{Le contexte de l'étude}

De 1983 à 1998, l'Iemvt (Maisons-Alfort, France) et l'Isra (Dakar, Sénégal) ont conduit un projet de suivi individuel des ovins et des caprins du Sénégal dans leur système d'élevage (programme Ppr) dans le but d'approfondir les connaissances sur les pratiques d'élevage traditionnel, d'identifier les principales pathologies des petits ruminants et d'étudier leurs performances zootechniques. Le passage régulier des techniciens dans les troupeaux a permis de recueillir des informations démographiques, sanitaires et zootechniques sur les animaux. La volonté affirmée de ce programme de ne pas modifier la conduite de l'élevage rendait le suivi des animaux totalement dépendant de la gestion traditionnelle du troupeau par l'éleveur. De 1992 à 1994, dans le cadre de ce projet, une étude sur le parasitisme gastro-intestinal a été conduite sur des animaux qui faisaient déjà partie du suivi. Des coproscopies et des prélèvements de sang ont été réalisés au même moment chez des femelles et chez leurs jeunes. Deux régions différentes ont été étudiées : la zone de Louga au nord du Sénégal (années 1992 et 1993), et la zone de Kolda au sud (1993 et 1994).

Au Sénégal, comme dans de nombreux pays en développement, la gestion des troupeaux est dictée à tout moment par les besoins financiers et socioculturels de l'éleveur et de sa famille. Ainsi, les ventes de jeunes animaux sont importantes - soit à l'occasion d'une fête religieuse, soit parce que l'éleveur a un besoin d'argent immédiat - et fluctuantes au cours de l'année. Il était donc difficile de savoir a priori si les animaux sélectionnés allaient être conservés les années suivantes. C'est pour cette raison que les prélèvements ont été réalisés simultanément chez les mères et chez leurs jeunes alors que, théoriquement, il aurait fallu mesurer les deux premières générations d'animaux au même âge, jeune ou adulte. Il était également difficile d'étudier les animaux pendant une période de temps relativement longue. Ainsi le nombre d'individus examinés pendant deux années successives a été faible.

Une autre difficulté liée au recueil de données en milieu traditionnel est la petite taille des élevages. L'effectif moyen par troupeau était de 3 animaux à Kolda et de 4 à Louga, avec des tailles de troupeaux pouvant aller de 2 à 30 animaux (figure 1).

\section{Les caractéristiques du milieu et les pratiques d'élevage}

Les deux régions étudiées sont différentes, tant du point de vue climatique que zootechnique. Sous un climat de type sahélien, la région de Louga, située au nord du Sénégal, est caractérisée par une longue saison sèche et une saison des pluies de juillet à septembre avec des précipitations de l'ordre de 200 à $300 \mathrm{~mm}$. Dans cette zone, 60 élevages répartis dans 15 villages ont été choisis pour le recueil de données. L'étude a concerné 193 caprins et 184 ovins en 1992, 249 caprins et 357 ovins en 1993. La zone de Kolda située en Casamance, en climat soudano-guinéen, est caractérisée par une végétation beaucoup plus fournie (savane boisée constituée de graminées vivaces). La saison des pluies s'étale de juin à octobre avec des précipitations atteignant $950 \mathrm{~mm}$. Quatrevingts élevages répartis dans 25 villages y ont été suivis pour le recueil des données de parasitologie. Les effectifs étaient de 322 caprins et 349 ovins en 1993, 428 caprins et 297 ovins en 1994.

Les pratiques d'élevage ont été décrites par Faugère et coll. (15, 16), Moulin (25) et Moulin et coll. (26). La principale caractéristique de ces pratiques est leur grande variabilité. Selon la région, l'ethnie et le village auquel appartient l'éleveur, la conduite des troupeaux peut être très différente.

A Louga, les deux ethnies principales sont les Wolofs et les Peuls. Les ovins de cette région sont des Peul-Peul, des Touabir (race généralement réservée à l'élevage de case) ou des Waralé (croisement Peul-Peul x Touabir) ; tous les caprins sont de race sahélienne. Les Peuls sont généralement propriétaires d'ovins et de caprins, ces derniers fournissant du lait. Hors saison des cultures, les animaux sevrés sont laissés en divagation aux alentours des villages et ils ont accès aux résidus de récolte et à la végétation naturelle de type savane (graminées annuelles). La nuit, les animaux sont placés dans des parcs ou sous des abris plus ou moins sophistiqués à l'intérieur de la concession - qui regroupe l'ensemble des habitations d'une même famille. Pendant la saison des cultures, les animaux sont gardés par les enfants ou conduits en troupeaux villageois sous la responsabilité d'un berger. Les Wolofs possèdent généralement des troupeaux composés uniquement d'ovins, et pratiquent un élevage plus intensif que les Peuls : les animaux sont conduits sur parcours pendant la journée, ils bénéficient d'un apport alimentaire pendant la saison sèche et le lait fourni par les brebis est prélevé en quantité importante.

A Kolda, pendant la saison des cultures, les Peuls — qui constituent la principale ethnie de cette partie du pays — attachent leurs animaux (ovins Djallonké et chèvres guinéennes) en bordure des champs. Dès la fin des récoltes, les animaux exploitent les résidus de cultures. Au fur et à mesure que la saison sèche avance, les ovins descendent dans les bas-fonds rizicoles et les caprins se nourrissent de la végétation des sous-bois sur les plateaux. Pendant la saison des pluies, les animaux sont attachés en bordure des champs ou conduits sur parcours, mais cette possibilité est beaucoup moins fréquente et réservée aux troupeaux de plus grande taille. 
La reproduction n'est pas maîtrisée et les pratiques actuelles (divagation des animaux autour des villages, conduite sur parcours en troupeaux collectifs, exploitation importante des mâles pour la viande) ne permettent pas d'identifier les paternités. Certains éleveurs louent ou achètent un mâle reproducteur pour leur troupeau, ou encore gardent un mâle du troupeau (choisi d'après sa conformation) pour la reproduction, les autres étant vendus ou castrés. Mais dans la grande majorité des cas, les mâles sont vendus pour la viande et la reproduction est alors assurée par les jeunes mâles de l'année précédente ou par un animal d'un troupeau voisin. En ce qui concerne la réforme et le remplacement des femelles, les éleveurs tiennent généralement compte de leurs performances de reproduction.

\section{Le protocole d'étude}

En raison des différences importantes entre types génétiques, systèmes de conduite et conditions climatiques, les analyses des deux régions et des deux espèces ont été conduites séparément. Les prélèvements fécaux ont été faits à trois reprises chaque année : en juillet-août (P1), en octobre-novembre (P2) et en décembre-janvier (P3). Le nombre d'Opg a été déterminé par la technique de McMaster, à partir de selles formolées pour les années 1992 et 1993, à partir de selles fraîches pour l'année 1994 (grâce à l'installation d'un laboratoire à Kolda). Les prélèvements de sang ont été réalisés lors du premier et du dernier passage dans les troupeaux : juillet-août (P1) et décembre-janvier (P3). Les animaux examinés en juillet-août l'ont été également les fois suivantes, sauf pour une minorité d'entre eux, vendus ou morts. Un petit nombre de femelles a pu être examiné pendant deux années successives. Tous les animaux étaient vaccinés contre la peste des petits ruminants et la pasteurellose. En 1994, les animaux de Kolda ont été traités à l'albendazole entre le deuxième et le troisième prélèvement.

Pour les raisons pratiques déjà évoquées, les prélèvements ont été réalisés au même moment sur les femelles et leurs produits. Cette approche sous-entend que les mécanismes de résistance sont identiques tout au long de la vie de l'animal. L'âge des jeunes animaux examinés était compris entre trois et huit mois, donc à la limite pour les animaux les plus jeunes il pouvait s'agir d'une première infestation. Or les très jeunes animaux n'ayant jamais été en contact avec des parasites ne possèdent pas encore la capacité à se défendre contre une infestation, les mécanismes de résistance se mettant en place progressivement pendant les premiers mois de leur vie à l'occasion de stimulations antigéniques par contact avec des parasites $(17,29)$. La vitesse à laquelle la résistance est acquise dépend du génotype de l'hôte (17). Pourtant Bishop et coll. (8) commencent à observer une variabilité génétique chez des agneaux à partir de trois mois. D'autre part, certaines études montrent que la corrélation mère-jeune pour le nombre d'Opg serait comprise entre $0,58(11,24)$ et $0,70(24)$. Ces résultats supposent que le déterminisme génétique de la résistance n'est pas totalement identique chez les jeunes et chez les adultes. Cependant, dans notre étude les distributions, les variances et les répétabilités étaient semblables chez les mères et chez les jeunes pour le caractère nombre d'Opg ; il y aurait néanmoins une petite différence, non significative, pour les variances de l'hématocrite entre mères et jeunes. D'autre part, la structure des données nous a imposé de considérer que le nombre d'Opg représentait le même caractère chez les mères et chez les jeunes de façon à bien valoriser l'information à l'aide d'un modèle animal. En réalité, le paramètre estimé par la relation mère-descendant correspondrait au produit de la corrélation génétique et de la racine carrée de chacune des héritabilités mère et jeune. Toutefois nous avons choisi de parler d'héritabilité puisque nous avons considéré que les caractères étaient les mêmes quel que soit l'âge des animaux. Compte tenu des résultats de corrélation obtenus par Bouix et coll. (11) et Morris et coll. (24), la valeur d'héritabilité obtenue dans ce travail par l'étude de la relation mère-jeune pour le caractère nombre d'Opg a été une estimation de la limite inférieure de la vraie valeur de l'héritabilité.

\section{Les analyses statistiques}

Les variables nombre d'Opg et hématocrite ont été analysées. La variable nombre d'Opg n'était pas distribuée selon une loi normale. La fonction logarithmique est souvent utilisée pour normaliser cette variable $(7,20,29)$. La transformation suivante $\left[\log _{10}(\right.$ nombre d'Opg +25$\left.)\right]$ a été appliquée aux données, après avoir vérifié que la distribution ainsi obtenue n'était pas significativement différente d'une loi normale ; la valeur 25 est due au fait qu'il n'est pas possible de détecter moins de $50 \mathrm{Opg}$. Le poids des jeunes à la date du prélèvement a été estimé à partir des pesées réalisées dans le cadre du suivi des animaux dans leur milieu d'élevage (programme Ppr) et contenues dans une base de données.

Dans un premier temps, les principaux facteurs environnementaux susceptibles d'influencer les caractères étudiés ont été identifiés et testés par des analyses de variance (modèles à effets fixés) à l'aide de la procédure Glm du logiciel Sas (30). Dans une première série d'analyses, un effet troupeau hiérarchisé dans l'effet village a été considéré. Cependant, le nombre de couples mère-jeune examinés par troupeau ayant été généralement faible (figure 1), la plupart des estimations obtenues étaient biaisées, les biais ayant résulté d'une confusion entre l'effet troupeau et l'effet animal. Nous avons alors choisi le village comme unité d'élevage, bien qu'il s'agisse d'une approximation. En effet, chaque éleveur possède ses propres pratiques d'élevage et celles-ci ne sont pas forcément

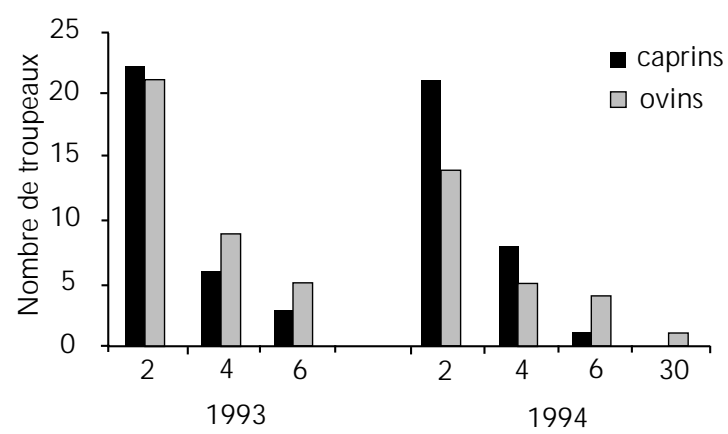

Nombre d'individus (mères + jeunes) examinés par troupeau Zone de Kolda

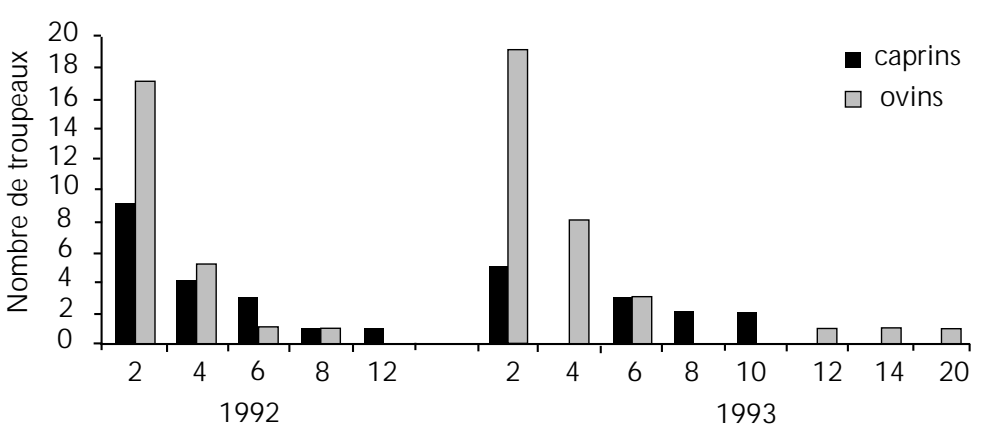

Nombre d'individus (mères + jeunes) examinés par troupeau Zone de Louga

Figure 1 : effectifs par troupeau. 
concordantes au sein d'un même village. La prise en compte d'un effet village en tant qu'unité d'élevage permettait néanmoins de tenir compte des caractéristiques environnementales (microclimat, type de pâturage, disponibilité en eau... ) qui présentent une certaine homogénéité pour un village donné. Une meilleure correction aurait pu être apportée en regroupant les troupeaux sur des critères mode de conduite (animaux conduits sur parcours en troupeaux collectifs, animaux laissés en divagation) et pratiques d'élevage (complémentation, traite... ). Toutefois ces informations n'étaient pas disponibles.

Les facteurs significatifs (testés dans les analyses de variance) ont été retenus et pris comme effets fixés dans les modèles mixtes pour les analyses ultérieures.

La répétabilité, qui mesure la ressemblance entre performances successives d'un individu, a été estimée par un modèle mixte pour les mères et les jeunes pris séparément à l'aide de la procédure Varcomp de Sas (30) ou simultanément à l'aide du logiciel Vce (28). L'estimation de la répétabilité avec ce logiciel n'étant possible que lorsque le modèle inclut une composante génétique, la procédure Varcomp a été utilisée pour les analyses concernant les mères ou les jeunes considérés séparément. Les analyses ont été faites intra et entre années. L'héritabilité a été estimée à l'aide d'un modèle animal (logiciel $V c e$ ). Les paternités n'étaient pas connues et les seules relations de parenté disponibles étaient les relations mère-descendants et demi-frères de mère, dans le cas de portées multiples. Les modèles mixtes utilisés pour l'analyse des caractères nombre d'Opg et hématocrite ont été les suivants :

\section{Chez les mères :}

$$
\mathrm{Y}_{\mathrm{ijklmn}}=\mu+\mathrm{A}_{\mathrm{i}}+\mathrm{P}_{\mathrm{i}}+\mathrm{tpy}_{\mathrm{jkl}}+\mathrm{b}_{\mathrm{m}}+\mathrm{r}_{\mathrm{n}}+\mathrm{E}_{\mathrm{ijklmn}}
$$

où $Y_{i j k l m n}$ est la performance de la mère $i$, du village $j$,

à la saison $\mathrm{k}$ de l'année $1, \mathrm{~d}$ 'âge $\mathrm{m}$ et de stade de lactation $\mathrm{n}$;

$\mu$ est la moyenne générale de la population ;

$\mathrm{A}_{\mathrm{i}}$ est la valeur génétique de la mère $\mathrm{i}$;

$\mathrm{P}_{\mathrm{i}}$ est l'effet aléatoire d'environnement permanent entre les performances successives de la mère $\mathrm{i}$ (cet effet permet d'estimer la répétabilité des caractères) ;

tpy $_{\mathrm{jkl}}$ est la combinaison village*saison*année (effet fixé) ;

$\mathrm{b}_{\mathrm{m}}$ est l'effet fixé âge ;

$\mathrm{r}_{\mathrm{n}}$ est l'effet fixé stade de lactation ;

$\mathrm{E}_{\mathrm{ijklmn}}$ est l'effet résiduel.

\section{Chez les jeunes :}

$$
\mathrm{Y}_{\mathrm{ijklmn}}=\mu+\mathrm{A}_{\mathrm{i}}+\mathrm{P}_{\mathrm{i}}+\mathrm{tpy}_{\mathrm{jkl}}+\mathrm{b}_{\mathrm{m}}+\mathrm{s}_{\mathrm{n}}+\mathrm{E}_{\mathrm{ijklmn}}
$$

où $Y_{i j k l m n}$ est la performance de l'animal $i$, du village $j$,

à la saison $\mathrm{k}$ de l'année $\mathrm{l}$, d'âge $\mathrm{m}$ et de sexe $\mathrm{n}$;

$\mu$ est la moyenne générale de la population;

$\mathrm{A}_{\mathrm{i}}$ est la valeur génétique de l'individu i ;

$\mathrm{P}_{\mathrm{i}}$ est l'effet aléatoire d'environnement permanent entre les performances successives de l'individu i ;

tpy $_{\mathrm{jkl}}$ est la combinaison village*saison*année (effet fixé);

$\mathrm{b}_{\mathrm{m}}$ est l'effet fixé âge ;

$\mathrm{s}_{\mathrm{n}}$ est l'effet fixé sexe ;

$\mathrm{E}_{\mathrm{ijklmn}}$ est la résiduelle.

Dans un premier temps, le facteur mode de naissance a été testé (dans les modèles à effets fixés), mais il a été trouvé non significatif dans les deux zones et pour les deux espèces, pour les caractères nombre d'Opg et hématocrite ; il n'a donc pas été conservé par la suite. Le nombre de villages variait entre 7 et 12 pour la zone de Louga et entre 17 et 21 pour la zone de Kolda, selon l'année et l'espèce considérées. L'effet saison correspond à la saison à laquelle ont été réalisés les trois prélèvements : début, milieu ou fin de la saison des pluies. La combinaison village*saison*année a été codée et prise en compte séparément pour les jeunes et pour les mères. Pour les effets de l'âge, des analyses préliminaires ont été réalisées. En tenant compte des valeurs estimées obtenues, l'âge des mères a été réparti en trois classes : inférieur à 2 ans, de 2 à 5 ans, supérieur à 5 ans, de même que l'âge des jeunes : inférieur à 4 mois, de 4 à 7 mois, supérieur à 7 mois. Plusieurs auteurs $(3,13$, 18) ont montré que le nombre d'Opg augmentait significativement chez les femelles à la fin de la gestation et pendant l'allaitement (phénomène appelé periparturient rise), consécutivement à une réduction des défenses immunitaires. Un effet stade de lactation a alors été ajouté dans le modèle. Il comprend trois niveaux, selon la durée de l'intervalle de temps entre le prélèvement et la mise bas qui le précède : femelles en début de lactation pour un intervalle inférieur à 2,5 mois, femelles en fin de lactation pour un intervalle compris entre 2,5 et 4,5 mois, et femelles non lactantes pour un intervalle supérieur à 5 mois. Chez les jeunes, le modèle comprend un effet sexe.

Les analyses (modèles mixtes) ont été réalisées pour chaque prélèvement (P1, P2, P3), pour plusieurs prélèvements simultanément $(\mathrm{P} 1+\mathrm{P} 2, \mathrm{P} 1+\mathrm{P} 3, \mathrm{P} 1+\mathrm{P} 2+\mathrm{P} 3)$, puis pour des prélèvements différents chez les mères et chez les jeunes (M1/J2, M2/J1, M1/J3, $\mathrm{M} 3 / \mathrm{J} 1, \mathrm{M} 2 / \mathrm{J} 3, \mathrm{M} 3 / \mathrm{J} 2$ ), de façon à étudier la robustesse de la relation entre les mères et les jeunes à des prélèvements différents. L'effet fixé prélèvement et l'effet aléatoire d'environnement permanent présentés dans les modèles mixtes ci-dessus concernaient les échantillons de données regroupant plusieurs prélèvements.

En plus du modèle précédent qui comprenait les effets fixés décrits précédemment, un effet animal et un effet d'environnement permanent (modèle 1), deux modèles mixtes supplémentaires ont été testés pour les échantillons contenant plusieurs prélèvements. Ces deux modèles comprenaient un effet aléatoire, de façon à essayer d'évaluer l'importance d'effets environnementaux ignorés en l'absence d'effet fixé troupeau. L'un était un effet aléatoire d'environnement commun pour le couple mère-jeune pour un prélèvement donné (modèle 2). L'autre était un effet aléatoire d'environnement commun entre les animaux appartenant au même troupeau pour un prélèvement donné (modèle 3 ).

Les corrélations génétiques et phénotypiques entre caractères n'ont pas pu être estimées correctement avec Vce. Les corrélations phénotypiques ont été obtenues à partir des résidus d'une analyse de variance à l'aide de la procédure Corr de Sas (30). Les facteurs pris en compte dans l'analyse de variance étaient ceux cités précédemment. Un facteur mode de naissance a été ajouté pour le poids des jeunes, car il avait un effet significatif sur ce caractère.

\section{RESU LTATS}

\section{Prévalence des parasites et niveau d'infestation}

Les œufs de parasites identifiés dans les coproscopies étaient en majorité des œufs de strongles. Des cultures fécales ont montré que parmi les strongles, les principaux genres étaient Haemonchus (40 p. 100) avec une prédominance d'Haemonchus contortus (espèce hématophage), Trichostrongylus (32 p. 100) et Esophagostomum (15 p. 100).

Les moyennes et les variances résiduelles ont été estimées à l'aide d'analyses de variance pour chaque prélèvement chez les mères et chez les jeunes. Les facteurs considérés étaient le village, l'âge et le sexe pour les jeunes, le village, l'âge et le stade de lactation pour les mères. Ces moyennes et ces variances sont présentées dans les tableaux I et II. 
Tableau I

Estimation des moyennes et des variances résiduelles obtenues à l'aide d'une analyse de variance (modèle à effets fixés) pour les caractères $\log _{10}$ (nombre d'Opg +25 ) et hématocrite pour chaque prélèvement chez les mères et les jeunes de la zone de suivi de Louga. Les effets du modèle sont le village, l'âge et le sexe pour les jeunes, le village, l'âge et le stade de lactation pour les mères

\begin{tabular}{|c|c|c|c|c|c|}
\hline & & Caprins 1992 & Caprins 1993 & O vins 1992 & O vins 1993 \\
\hline \multicolumn{6}{|l|}{ Mères } \\
\hline Nombre d'Opg (1) & $\begin{array}{l}\text { Effectif } \\
\text { Moyenne } \\
\text { Variance } \\
\mathrm{R}^{2}\end{array}$ & $\begin{array}{l}105 \\
2,01 \\
0,20 \\
0,12\end{array}$ & $\begin{array}{l}130 \\
2,18 \\
0,26 \\
0,24\end{array}$ & $\begin{array}{c}96 \\
2,05 \\
0,34 \\
0,14\end{array}$ & $\begin{array}{l}184 \\
2,18 \\
0,30 \\
0,12\end{array}$ \\
\hline Nombre d'Opg (2) & $\begin{array}{l}\text { Effectif } \\
\text { Moyenne } \\
\text { Variance } \\
\mathrm{R}^{2}\end{array}$ & $\begin{array}{c}94 \\
2,31 \\
0,21 \\
0,06\end{array}$ & $\begin{array}{c}60 \\
1,86 \\
0,16 \\
0,24\end{array}$ & $\begin{array}{c}92 \\
2,31 \\
0,28 \\
0,06\end{array}$ & $\begin{array}{l}128 \\
1,91 \\
0,20 \\
0,35\end{array}$ \\
\hline Nombre d'Opg (3) & $\begin{array}{l}\text { Effectif } \\
\text { Moyenne } \\
\text { Variance } \\
\mathrm{R}^{2}\end{array}$ & $\begin{array}{c}54 \\
2,66 \\
0,33 \\
0,23\end{array}$ & $\begin{array}{c}58 \\
1,79 \\
0,13 \\
0,28\end{array}$ & $\begin{array}{c}70 \\
2,41 \\
0,40 \\
0,28\end{array}$ & $\begin{array}{l}116 \\
1,75 \\
0,12 \\
0,37\end{array}$ \\
\hline H ématocrite (1) & $\begin{array}{l}\text { Effectif } \\
\text { Moyenne } \\
\text { Variance } \\
\mathrm{R}^{2}\end{array}$ & $\begin{array}{l}104 \\
27,3 \\
20,3 \\
0,21\end{array}$ & $\begin{array}{l}128 \\
30,0 \\
27,5 \\
0,08\end{array}$ & $\begin{array}{c}96 \\
27,4 \\
12,9 \\
0,30\end{array}$ & $\begin{array}{l}183 \\
31,5 \\
14,9 \\
0,36\end{array}$ \\
\hline Hématocrite (3) & $\begin{array}{l}\text { Effectif } \\
\text { M oyenne } \\
\text { Variance } \\
\mathrm{R}^{2}\end{array}$ & $\begin{array}{c}62 \\
28,8 \\
19,9 \\
0,28\end{array}$ & $\begin{array}{c}59 \\
32,8 \\
29,9 \\
0,38\end{array}$ & $\begin{array}{c}80 \\
28,5 \\
26,8 \\
0,18\end{array}$ & $\begin{array}{l}117 \\
31,8 \\
19,1 \\
0,27\end{array}$ \\
\hline \multicolumn{6}{|l|}{ Jeunes } \\
\hline Nombre d'O pg (1) & $\begin{array}{l}\text { Effectif } \\
\text { Moyenne } \\
\text { Variance } \\
\mathrm{R}^{2}\end{array}$ & $\begin{array}{c}88 \\
2,14 \\
0,30 \\
0,26\end{array}$ & $\begin{array}{l}119 \\
2,59 \\
0,31 \\
0,21\end{array}$ & $\begin{array}{c}88 \\
1,96 \\
0,33 \\
0,28\end{array}$ & $\begin{array}{l}173 \\
2,39 \\
0,38 \\
0,27\end{array}$ \\
\hline Nombre d'Opg (2) & $\begin{array}{l}\text { Effectif } \\
\text { M oyenne } \\
\text { Variance } \\
\mathrm{R}^{2}\end{array}$ & $\begin{array}{c}82 \\
2,62 \\
0,27 \\
0,12\end{array}$ & $\begin{array}{c}85 \\
2,01 \\
0,18 \\
0,41\end{array}$ & $\begin{array}{c}81 \\
2,52 \\
0,35 \\
0,38\end{array}$ & $\begin{array}{l}138 \\
2,30 \\
0,41 \\
0,26\end{array}$ \\
\hline Nombre d'Opg (3) & $\begin{array}{l}\text { Effectif } \\
\text { Moyenne } \\
\text { Variance } \\
\mathrm{R}^{2}\end{array}$ & $\begin{array}{c}63 \\
2,77 \\
0,34 \\
0,37\end{array}$ & $\begin{array}{c}66 \\
1,64 \\
0,11 \\
0,28\end{array}$ & $\begin{array}{c}68 \\
2,85 \\
0,41 \\
0,20\end{array}$ & $\begin{array}{l}112 \\
1,71 \\
0,14 \\
0,15\end{array}$ \\
\hline Hématocrite (1) & $\begin{array}{l}\text { Effectif } \\
\text { Moyenne } \\
\text { Variance } \\
\mathrm{R}^{2}\end{array}$ & $\begin{array}{c}88 \\
28,0 \\
41,9 \\
0,31\end{array}$ & $\begin{array}{l}117 \\
32,0 \\
33,9 \\
0,29\end{array}$ & $\begin{array}{c}86 \\
29,4 \\
33,9 \\
0,18\end{array}$ & $\begin{array}{l}173 \\
33,3 \\
19,4 \\
0,34\end{array}$ \\
\hline H ématocrite (3) & $\begin{array}{l}\text { Effectif } \\
\text { Moyenne } \\
\text { Variance } \\
\mathrm{R}^{2}\end{array}$ & $\begin{array}{c}62 \\
27,7 \\
39,3 \\
0,47\end{array}$ & $\begin{array}{c}63 \\
32,4 \\
25,2 \\
0,53\end{array}$ & $\begin{array}{c}69 \\
28,3 \\
39,2 \\
0,18\end{array}$ & $\begin{array}{l}111 \\
33,1 \\
22,0 \\
0,27\end{array}$ \\
\hline
\end{tabular}

$1,2,3$ : numéro du prélèvement $; \mathrm{R}^{2}$ : coefficient de détermination

La moyenne des valeurs du $\log _{10}$ (nombre d'Opg + 25) sur les deux zones de suivi était de 2,35 chez les jeunes et 2,15 chez les mères, ce qui correspondait respectivement à 200 et 120 Opg. En moyenne, les taux d'infestation étaient semblables pour les deux espèces, mais légèrement supérieurs à Kolda. Les valeurs des coproscopies (en nombre d'Opg) variaient beaucoup selon l'échantillon considéré. Elles allaient de 20 à 1 200, avec une valeur très forte de 1600 Opg pour le deuxième prélèvement chez les agneaux de Kolda en 1994. Par rapport aux deux premiers prélèvements, le troisième prélèvement de Kolda en 1994, qui faisait suite au déparasitage, montrait un niveau d'infestation plus faible.

Pour les mesures d'infestation, les variances des mères et des jeunes étaient proches ; elles étaient légèrement différentes pour l'hématocrite, bien que ce ne soit pas significatif avec un test de 


\section{Tableau II}

Estimation des moyennes et des variances résiduelles obtenues à l'aide d'une analyse de variance (modèle à effets fixés) pour les caractères $\log _{10}$ (nombre d'Opg +25 ) et hématocrite pour chaque prélèvement chez les mères et les jeunes de la zone de suivi de Kolda. Les effets du modèle sont le village, l'âge et le sexe pour les jeunes, le village, l'âge et le stade de lactation pour les mères

\begin{tabular}{|c|c|c|c|c|c|}
\hline & & Caprins 1993 & Caprins 1994 & O vins 1993 & O vins 1994 \\
\hline \multicolumn{6}{|l|}{ Mères } \\
\hline Nombre d'O pg (1) & $\begin{array}{l}\text { Effectif } \\
\text { Moyenne } \\
\text { Variance } \\
\mathrm{R}^{2}\end{array}$ & $\begin{array}{l}199 \\
1,85 \\
0,16 \\
0,39\end{array}$ & $\begin{array}{l}178 \\
2,60 \\
0,23 \\
0,33\end{array}$ & $\begin{array}{l}207 \\
1,92 \\
0,19 \\
0,23\end{array}$ & $\begin{array}{l}233 \\
2,27 \\
0,32 \\
0,29\end{array}$ \\
\hline Nombre d'O pg (2) & $\begin{array}{l}\text { Effectif } \\
\text { Moyenne } \\
\text { Variance } \\
\mathrm{R}^{2}\end{array}$ & $\begin{array}{l}139 \\
2,05 \\
0,20 \\
0,18\end{array}$ & $\begin{array}{l}104 \\
2,87 \\
0,23 \\
0,30\end{array}$ & $\begin{array}{l}152 \\
2,04 \\
0,19 \\
0,23\end{array}$ & $\begin{array}{l}166 \\
2,67 \\
0,24 \\
0,24\end{array}$ \\
\hline Nombre d'Opg (3) & $\begin{array}{l}\text { Effectif } \\
\text { Moyenne } \\
\text { Variance } \\
\mathrm{R}^{2}\end{array}$ & $\begin{array}{c}99 \\
2,04 \\
0,15 \\
0,50\end{array}$ & $\begin{array}{c}71 \\
2,01 \\
0,14 \\
0,40\end{array}$ & $\begin{array}{l}112 \\
2,03 \\
0,18 \\
0,32\end{array}$ & $\begin{array}{l}136 \\
2,12 \\
0,18 \\
0,38\end{array}$ \\
\hline H ématocrite (1) & $\begin{array}{l}\text { Effectif } \\
\text { Moyenne } \\
\text { Variance } \\
\mathrm{R}^{2}\end{array}$ & $\begin{array}{l}197 \\
34,2 \\
21,0 \\
0,34\end{array}$ & $\begin{array}{l}175 \\
33,0 \\
45,9 \\
0,31\end{array}$ & $\begin{array}{l}201 \\
32,4 \\
17,9 \\
0,28\end{array}$ & $\begin{array}{l}230 \\
31,1 \\
32,8 \\
0,12\end{array}$ \\
\hline H ématocrite (3) & $\begin{array}{l}\text { Effectif } \\
\text { Moyenne } \\
\text { Variance } \\
\mathrm{R}^{2}\end{array}$ & $\begin{array}{c}98 \\
29,8 \\
33,6 \\
0,37\end{array}$ & $\begin{array}{c}66 \\
35,3 \\
20,5 \\
0,45\end{array}$ & $\begin{array}{l}118 \\
28,7 \\
14,9 \\
0,34\end{array}$ & $\begin{array}{l}125 \\
30,4 \\
16,8 \\
0,22\end{array}$ \\
\hline \multicolumn{6}{|l|}{ Jeunes } \\
\hline Nombre d'Opg (1) & $\begin{array}{l}\text { Effectif } \\
\text { Moyenne } \\
\text { Variance } \\
\mathrm{R}^{2}\end{array}$ & $\begin{array}{l}123 \\
2,14 \\
0,26 \\
0,27\end{array}$ & $\begin{array}{l}119 \\
2,59 \\
0,25 \\
0,38\end{array}$ & $\begin{array}{l}142 \\
2,09 \\
0,25 \\
0,33\end{array}$ & $\begin{array}{l}195 \\
2,56 \\
0,32 \\
0,20\end{array}$ \\
\hline Nombre d'Opg (2) & $\begin{array}{l}\text { Effectif } \\
\text { Moyenne } \\
\text { Variance } \\
\mathrm{R}^{2}\end{array}$ & $\begin{array}{l}108 \\
2,39 \\
0,32 \\
0,20\end{array}$ & $\begin{array}{l}106 \\
3,21 \\
0,22 \\
0,20\end{array}$ & $\begin{array}{l}129 \\
2,39 \\
0,24 \\
0,40\end{array}$ & $\begin{array}{l}170 \\
3,09 \\
0,23 \\
0,31\end{array}$ \\
\hline Nombre d'Opg (3) & $\begin{array}{l}\text { Effectif } \\
\text { Moyenne } \\
\text { Variance } \\
\mathrm{R}^{2}\end{array}$ & $\begin{array}{c}90 \\
2,14 \\
0,15 \\
0,25\end{array}$ & $\begin{array}{c}92 \\
1,89 \\
0,15 \\
0,31\end{array}$ & $\begin{array}{l}102 \\
2,21 \\
0,19 \\
0,28\end{array}$ & $\begin{array}{l}155 \\
2,25 \\
0,18 \\
0,34\end{array}$ \\
\hline H ématocrite (1) & $\begin{array}{l}\text { Effectif } \\
\text { Moyenne } \\
\text { Variance } \\
\mathrm{R}^{2}\end{array}$ & $\begin{array}{l}123 \\
38,3 \\
37,9 \\
0,32\end{array}$ & $\begin{array}{l}114 \\
39,1 \\
45,8 \\
0,30\end{array}$ & $\begin{array}{l}143 \\
34,8 \\
17,1 \\
0,31\end{array}$ & $\begin{array}{l}189 \\
34,1 \\
25,9 \\
0,23\end{array}$ \\
\hline H ématocrite (3) & $\begin{array}{l}\text { Effectif } \\
\text { Moyenne } \\
\text { Variance } \\
\mathrm{R}^{2}\end{array}$ & $\begin{array}{c}88 \\
27,7 \\
39,3 \\
0,47\end{array}$ & $\begin{array}{c}88 \\
32,4 \\
25,2 \\
0,53\end{array}$ & $\begin{array}{l}100 \\
27,7 \\
16,8 \\
0,41\end{array}$ & $\begin{array}{l}148 \\
30,1 \\
19,8 \\
0,36\end{array}$ \\
\hline
\end{tabular}

$1,2,3$ : numéro du prélèvement $; \mathrm{R}^{2}$ : coefficient de détermination

Bartlett. La variance résiduelle en nombre d'Opg (variable transformée) était comprise entre 0,11 et 0,41 . Au troisième prélèvement de la zone de Kolda, les variances étaient un peu plus faibles (de 0,14 à 0,19$)$ aussi bien chez les mères que chez les jeunes, ce qui peut s'expliquer par le traitement antiparasitaire appliqué entre le deuxième et le troisième prélèvement. Les variances de l'hématocrite étaient comprises entre 12,9 et 45,9. Aucun effet du déparasitage effectué juste avant le troisième prélèvement de Kolda n'a pu être mis en évidence pour ce caractère.

\section{Facteurs de variation des critères de résistance}

Les facteurs environnementaux testés avaient généralement une influence assez forte sur les caractères de résistance. Les coefficients de détermination $\left(\mathrm{R}^{2}\right)$ des modèles d'analyse étaient compris entre 0,20 et 0,30 . Le facteur village était toujours significatif pour les deux variables testées. La saison avait un effet significatif sur le nombre d'Opg. A Kolda, le niveau maximum d'infestation a été observé au deuxième prélèvement, c'est-à-dire en octobre- 
novembre. A Louga, le profil était différent selon l'année étudiée. En 1992, le niveau d'infestation mesuré en Opg augmentait au fur et à mesure de la saison des pluies, alors qu'en 1993 il était très élevé en début de saison des pluies puis diminuait pour les prélèvements suivants. Cette variation du nombre d'Opg pour l'année 1993 peut être expliquée par des précipitations relativement importantes dès le début de la saison des pluies.

En ce qui concerne le sexe, le nombre d'Opg était plus élevé et l'hématocrite plus faible chez les mâles que chez les femelles. L'âge des jeunes avait une influence sur la résistance au parasitisme : les animaux les plus jeunes étaient les plus infestés (plus grand nombre d'Opg), mais ils présentaient un taux de globules rouges dans le sang plus élevé. Chez les mères, le même résultat a été obtenu : les femelles de moins de deux ans avaient un nombre d'Opg et une valeur de l'hématocrite plus élevés. Le stade de lactation avait une incidence sur le niveau d'infestation mesuré en nombre d'Opg — d'autant plus élevé que le prélèvement avait été réalisé peu de temps après une gestation — mais n'en avait pas sur l'hématocrite, le facteur stade de lactation étant généralement non significatif pour ce critère.

\section{Estimation de la répétabilité}

Les répétabilités intra et entre années sont présentées dans les tableaux III pour le nombre d'Opg et IV pour l'hématocrite. Les répétabilités étaient généralement plus fortes à Louga qu'à Kolda. Lorsqu'elles étaient estimées simultanément chez les mères et les jeunes, les répétabilités intra-années variaient de 0,07 à 0,33 pour le nombre d'Opg et de 0,14 à 0,52 pour l'hématocrite, la moyenne des valeurs s'établissant respectivement à 0,19 et à 0,27 . Les valeurs obtenues pour les mères et les jeunes n'étaient pas très différentes. Elles étaient plus faibles entre années — de 0,11 à 0,16 pour le nombre d'Opg, de 0,20 à 0,30 pour l'hématocrite - mais avec des effectifs plus réduits.

\section{Tableau III}

Estimation des répétabilités intra et entre années (modèles mixtes) du $\log _{10}$ (nombre d'0 pg +25 ) pour les mères et les jeunes des zones de suivi de Louga et de Kolda. Analyses faites pour chacune des deux années d'études et pour deux années simultanément, avec le logiciel Sas (30) pour les mères et les jeunes séparément et avec le logiciel Vce (28) pour les mères et les jeunes simultanément

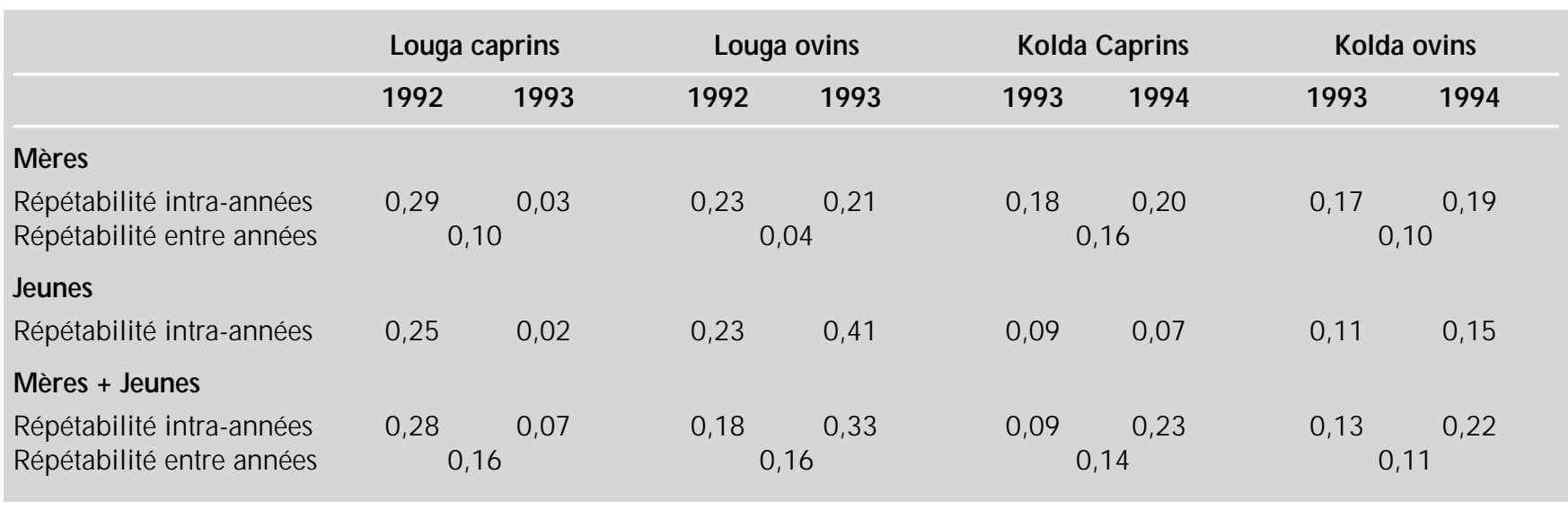

\section{Tableau IV}

Estimation des répétabilités intra et entre années (modèles mixtes) de l'hématocrite pour les mères et les jeunes des zones de suivi de Louga et de Kolda. Analyses faites pour chacune des deux années d'études et pour deux années simultanément, avec le logiciel Sas (30) pour les mères et les jeunes séparément et avec le logiciel Vce (28) pour les mères et les jeunes simultanément

\begin{tabular}{|c|c|c|c|c|c|c|c|c|}
\hline & \multicolumn{2}{|c|}{ Louga caprins } & \multicolumn{2}{|c|}{ Louga ovins } & \multicolumn{2}{|c|}{ Kolda Caprins } & \multicolumn{2}{|c|}{ Kolda ovins } \\
\hline & 1992 & 1993 & 1992 & 1993 & 1993 & 1994 & 1993 & 1994 \\
\hline \multicolumn{9}{|l|}{ Mères } \\
\hline Répétabilité intra-années & 0,50 & 0,23 & 0,38 & 0,25 & 0,23 & 0 & 0,18 & 0,35 \\
\hline Répétabilité entre années & \multicolumn{2}{|c|}{0,14} & \multicolumn{2}{|c|}{0,02} & \multicolumn{2}{|c|}{0,21} & \multicolumn{2}{|c|}{0,23} \\
\hline \multicolumn{9}{|l|}{ Jeunes } \\
\hline Répétabilité intra-années & 0,34 & 0,26 & 0,60 & 0,27 & 0,08 & 0,04 & 0,24 & 0,13 \\
\hline \multicolumn{9}{|l|}{ Mères + Jeunes } \\
\hline Répétabilité intra-années & 0,44 & 0,30 & 0,52 & 0,23 & 0,28 & 0,14 & 0,19 & 0,34 \\
\hline Répétabilité entre années & \multicolumn{2}{|c|}{0,30} & \multicolumn{2}{|c|}{0,31} & \multicolumn{2}{|c|}{0,20} & \multicolumn{2}{|c|}{0,26} \\
\hline
\end{tabular}




\section{Estimation de l'héritabilité}

Les héritabilités estimées sont présentées dans les tableaux $\mathrm{V}$ et VI. Les estimations étaient très variables selon l'échantillon étudié. Elles pouvaient atteindre des valeurs relativement élevées lorsqu'elles concernaient un seul prélèvement, jusqu'à 0,94 pour les coproscopies en nombre d'Opg et 1,00 pour l'hématocrite. L'ajout d'un ou deux prélèvements supplémentaires a entraîné une diminution de l'héritabilité. La prise en compte de prélèvements différents chez la mère et le jeune a conduit à des héritabilités proches de zéro. D'après ces résultats, la ressemblance entre individus est plus élevée intra-prélèvement qu'entre prélèvements. Il semblerait exister une ressemblance d'origine environnementale entre la mère et son jeune liée au milieu qu'ils partagent, ou au contraire une dissemblance entre prélèvements réalisés à des époques différentes due à des effets environnementaux mal ajustés.
La ressemblance intra-prélèvement ou la dissemblance entre prélèvements pourrait être la conséquence d'une mauvaise prise en compte des facteurs de milieu, et notamment de l'effet troupeau. Pour vérifier ces hypothèses, deux modèles supplémentaires ont été testés : en plus des effets animal et environnement permanent (modèle 1), le modèle 2 comprenait un effet d'environnement commun à la mère et à ses jeunes (Env-com) et le modèle 3 un effet troupeau*prélèvement (troup*P).

Les effets Env-com et troup*P étaient généralement non nuls (tableau VII) et leur prise en compte dans le modèle a entraîné une diminution de la variance génétique (l'héritabilité était alors proche de zéro), ce qui allait dans le sens de l'hypothèse posée. Ces effets variaient entre 0,03 et 0,25 pour le nombre d'Opg et entre 0 et 0,38 pour l'hématocrite. Pour la plupart des échantillons analysés, la répétabilité se maintenait quel que soit le modèle utilisé.

\section{Tableau V}

Estimation de l'héritabilité et de la répétabilité (modèles mixtes) du $\log _{10}$ (nombre d'Opg +25 ) pour différents échantillons de données

\begin{tabular}{|c|c|c|c|c|c|c|c|c|c|}
\hline \multirow[t]{2}{*}{ Echantillons } & \multirow[t]{2}{*}{ Paramètres } & \multicolumn{2}{|c|}{ Louga caprins } & \multicolumn{2}{|c|}{ Louga ovins } & \multicolumn{2}{|c|}{ Kolda Caprins } & \multicolumn{2}{|c|}{ Kolda ovins } \\
\hline & & 1992 & 1993 & 1992 & 1993 & 1993 & 1994 & 1993 & 1994 \\
\hline P1 & $h^{2}$ & 0,57 & 0 & 0,31 & 0,20 & 0,06 & 0,38 & 0,27 & 0,52 \\
\hline P2 & $h^{2}$ & 0,29 & 0,26 & 0 & 0,43 & 0,54 & 0,26 & 0 & 0 \\
\hline P3 & $h^{2}$ & 0 & 0,03 & 0 & 0,34 & 0,16 & 0,94 & 0,26 & 0,76 \\
\hline $\mathrm{M} 1 / \mathrm{J} 2$ & $h^{2}$ & 0,45 & 0 & 0,08 & 0 & 0,05 & 0 & 0 & 0 \\
\hline M 2/J1 & $h^{2}$ & 0,05 & 0 & 0,03 & 0,18 & 0 & 0,10 & 0 & 0,33 \\
\hline $\mathrm{M} 1 / \mathrm{J} 3$ & $h^{2}$ & 0 & 0,08 & 0 & 0,03 & 0,36 & 0,36 & 0 & 0 \\
\hline M 3/J1 & $h^{2}$ & 0 & 0 & 0 & 0,05 & 0,25 & 0,56 & 0 & 0,21 \\
\hline M 2/J3 & $h^{2}$ & 0,09 & 0,40 & 0 & 0,44 & 0 & 0,32 & 0,56 & 0,31 \\
\hline M 3/J2 & $h^{2}$ & 0 & 0,45 & 0 & 0,06 & 0,02 & 0,10 & 0 & 0,48 \\
\hline $\mathrm{P} 1+\mathrm{P} 2$ & $\begin{array}{c}h^{2} \\
R\end{array}$ & $\begin{array}{l}0,32 \\
0,43\end{array}$ & $\begin{array}{c}0 \\
0,07\end{array}$ & $\begin{array}{l}0,14 \\
0,14\end{array}$ & $\begin{array}{l}0,18 \\
0,35\end{array}$ & $\begin{array}{l}0,10 \\
0,15\end{array}$ & $\begin{array}{l}0,17 \\
0,21\end{array}$ & $\begin{array}{c}0 \\
0,14\end{array}$ & $\begin{array}{l}0,28 \\
0,37\end{array}$ \\
\hline $\mathrm{P} 1+\mathrm{P} 2+\mathrm{P} 3$ & $\begin{array}{c}h^{2} \\
R\end{array}$ & $\begin{array}{c}0 \\
0,28\end{array}$ & $\begin{array}{l}0,05 \\
0,07\end{array}$ & $\begin{array}{c}0 \\
0,18\end{array}$ & $\begin{array}{l}0,14 \\
0,33\end{array}$ & $\begin{array}{l}0,09 \\
0,09\end{array}$ & $\begin{array}{l}0,12 \\
0,23\end{array}$ & $\begin{array}{c}0 \\
0,13\end{array}$ & $\begin{array}{l}0,22 \\
0,22\end{array}$ \\
\hline
\end{tabular}

\section{Tableau VI}

Estimation de I'héritabilité et de la répétabilité (modèles mixtes) de I'hématocrite pour différents échantillons de données

\begin{tabular}{|c|c|c|c|c|c|c|c|c|c|}
\hline \multirow[t]{2}{*}{ Echantillons } & \multirow[t]{2}{*}{ Paramètres } & \multicolumn{2}{|c|}{ Louga caprins } & \multicolumn{2}{|c|}{ Louga ovins } & \multicolumn{2}{|c|}{ Kolda Caprins } & \multicolumn{2}{|c|}{ Kolda ovins } \\
\hline & & 1992 & 1993 & 1992 & 1993 & 1993 & 1994 & 1993 & 1994 \\
\hline P1 & $h^{2}$ & 0,05 & 0,65 & 0,02 & 0,36 & 0,11 & 0,76 & 0,23 & 0,42 \\
\hline P3 & $h^{2}$ & 0,26 & 0,40 & 0 & 0,72 & 1,00 & 0 & 0,62 & 1,00 \\
\hline$M 1 / J 3$ & $h^{2}$ & 0,45 & 0 & 0 & 0,40 & 0 & 0,29 & 0,49 & 0,12 \\
\hline M 3/J1 & $h^{2}$ & 0 & 0,19 & 0 & 0,03 & 0,08 & 0 & 0,83 & 0,32 \\
\hline $\mathrm{P} 1+\mathrm{P} 3$ & $\begin{array}{c}\mathrm{h}^{2} \\
\mathrm{R}\end{array}$ & $\begin{array}{l}0,16 \\
0,44\end{array}$ & $\begin{array}{l}0,30 \\
0,30\end{array}$ & $\begin{array}{c}0 \\
0,52\end{array}$ & $\begin{array}{l}0,23 \\
0,23\end{array}$ & $\begin{array}{l}0,05 \\
0,28\end{array}$ & $\begin{array}{l}0,14 \\
0,14\end{array}$ & $\begin{array}{l}0,19 \\
0,19\end{array}$ & $\begin{array}{l}0,34 \\
0,34\end{array}$ \\
\hline
\end{tabular}




\section{Tableau VII}

Estimation des paramètres génétiques (modèles mixtes) du $\log _{10}$ (nombre d'O pg +25 ) et de I'hématocrite pour différents modèles d'analyse (échantillon $\mathrm{P} 1+\mathrm{P} 2+\mathrm{P} 3$ pour le nombre d'O pg

et $P 1+P 3$ pour l'hématocrite)

\begin{tabular}{|c|c|c|c|c|c|c|c|c|c|}
\hline \multirow[t]{2}{*}{ Echantillons } & \multirow[t]{2}{*}{ Paramètres } & \multicolumn{2}{|c|}{ Louga caprins } & \multicolumn{2}{|c|}{ Louga ovins } & \multicolumn{2}{|c|}{ Kolda Caprins } & \multicolumn{2}{|c|}{ Kolda ovins } \\
\hline & & 1992 & 1993 & 1992 & 1993 & 1993 & 1994 & 1993 & 1994 \\
\hline \multicolumn{10}{|c|}{ Nombre d'O pg } \\
\hline Modèle 1 & $\begin{array}{c}h^{2} \\
R\end{array}$ & $\begin{array}{c}0 \\
0,28\end{array}$ & $\begin{array}{l}0,05 \\
0,07\end{array}$ & $\begin{array}{c}0 \\
0,18\end{array}$ & $\begin{array}{l}0,14 \\
0,33\end{array}$ & $\begin{array}{l}0,09 \\
0,09\end{array}$ & $\begin{array}{l}0,12 \\
0,23\end{array}$ & $\begin{array}{c}0 \\
0,13\end{array}$ & $\begin{array}{l}0,22 \\
0,22\end{array}$ \\
\hline Modèle 2 & $\begin{array}{c}h^{2} \\
R \\
\text { Env-com }\end{array}$ & $\begin{array}{c}0 \\
0,30 \\
0,25\end{array}$ & $\begin{array}{c}0 \\
0,07 \\
0,05\end{array}$ & $\begin{array}{c}0 \\
0,18 \\
0,09\end{array}$ & $\begin{array}{l}0,03 \\
0,34 \\
0,14\end{array}$ & $\begin{array}{c}0 \\
0,10 \\
0,18\end{array}$ & $\begin{array}{c}0 \\
0,25 \\
0,23\end{array}$ & $\begin{array}{c}0 \\
0,14 \\
0,06\end{array}$ & $\begin{array}{l}0,15 \\
0,20 \\
0,12\end{array}$ \\
\hline Modèle 3 & $\begin{array}{c}h^{2} \\
R \\
\text { Troup*P }\end{array}$ & $\begin{array}{c}0 \\
0,28 \\
0,09\end{array}$ & $\begin{array}{c}0 \\
0,07 \\
0,12\end{array}$ & $\begin{array}{c}0 \\
0,15 \\
0,11\end{array}$ & $\begin{array}{l}0,10 \\
0,32 \\
0,07\end{array}$ & $\begin{array}{l}0,07 \\
0,09 \\
0,03\end{array}$ & $\begin{array}{l}0,05 \\
0,27 \\
0,16\end{array}$ & $\begin{array}{c}0 \\
0,14 \\
0,07\end{array}$ & $\begin{array}{l}0,09 \\
0,18 \\
0,24\end{array}$ \\
\hline \multicolumn{10}{|l|}{ Hématocrite } \\
\hline Modèle 1 & $\begin{array}{c}\mathrm{h}^{2} \\
\mathrm{R}\end{array}$ & $\begin{array}{l}0,16 \\
0,44\end{array}$ & $\begin{array}{l}0,30 \\
0,30\end{array}$ & $\begin{array}{c}0 \\
0,52\end{array}$ & $\begin{array}{l}0,23 \\
0,23\end{array}$ & $\begin{array}{l}0,05 \\
0,28\end{array}$ & $\begin{array}{l}0,14 \\
0,14\end{array}$ & $\begin{array}{l}0,19 \\
0,19\end{array}$ & $\begin{array}{l}0,34 \\
0,34\end{array}$ \\
\hline Modèle 2 & $\begin{array}{c}h^{2} \\
R \\
\text { Env-com }\end{array}$ & $\begin{array}{c}0,16 \\
0,44 \\
0\end{array}$ & $\begin{array}{c}0 \\
0,26 \\
0,26\end{array}$ & $\begin{array}{c}0 \\
0,52 \\
0\end{array}$ & $\begin{array}{l}0,20 \\
0,20 \\
0,13\end{array}$ & $\begin{array}{c}0,05 \\
0,28 \\
0\end{array}$ & $\begin{array}{c}0 \\
0,13 \\
0,19\end{array}$ & $\begin{array}{c}0,19 \\
0,19 \\
0\end{array}$ & $\begin{array}{l}0,31 \\
0,31 \\
0,07\end{array}$ \\
\hline Modèle 3 & $\begin{array}{c}h^{2} \\
\mathrm{R} \\
\text { Troup*P }\end{array}$ & $\begin{array}{c}0 \\
0,35 \\
0,21\end{array}$ & $\begin{array}{c}0 \\
0,09 \\
0,38\end{array}$ & $\begin{array}{c}0 \\
0,51 \\
0,06\end{array}$ & $\begin{array}{l}0,17 \\
0,17 \\
0,13\end{array}$ & $\begin{array}{c}0 \\
0,25 \\
0,13\end{array}$ & $\begin{array}{l}0,10 \\
0,10 \\
0,08\end{array}$ & $\begin{array}{l}0,09 \\
0,09 \\
0,12\end{array}$ & $\begin{array}{l}0,02 \\
0,20 \\
0,36\end{array}$ \\
\hline
\end{tabular}

Modèle $1:$ un effet aléatoire animal + un effet d'environnement permanent

Modèle 2 : modèle $1+$ un effet d'environnement commun entre la mère et son jeune pour un prélèvement donné (Env-com)

Modèle 3 : modèle 1 + un effet aléatoire d'environnement commun entre les individus d'un même troupeau pour un prélèvement donné (troup*P)

$\mathrm{h}^{2}:$ héritabilité

$\mathrm{R}$ : répétabilité

Env-com : part de variance due à l'effet d'environnement commun entre mère et jeune

Troup*P : part de variance due à l'effet d'environnement commun entre animaux d'un même troupeau

\section{Estimation des corrélations phénotypiques}

Les corrélations phénotypiques entre caractères sont présentées dans les tableaux VIII et IX. Il existe une relation négative entre les caractères nombre d'Opg et hématocrite, excepté chez les mères pour le prélèvement qui a suivi le traitement anthelmintique (corrélation nulle au troisième prélèvement de Kolda en 1994). La corrélation était plus négative chez les jeunes $(-0,47$ à - 0,10$)$ que chez les mères $(-0,33$ à 0,03$)$. La corrélation entre les caractères nombre d'Opg et poids était très variable. Lorsque les analyses étaient faites sur un seul prélèvement, la taille de l'échantillon était très faible (12 individus pour le troisième prélèvement de Kolda en 1994). Pour une analyse sur deux années (en considérant tous les prélèvements), la corrélation variait entre - 0,35 et - 0,01 . La corrélation entre l'hématocrite et le poids était positive et relativement élevée : de 0,07 à 0,36 .

\section{DISCUSSION}

\section{Estimation de la répétabilité}

Les valeurs estimées de la répétabilité intra-années étaient plus élevées à Louga qu'à Kolda. La zone de Kolda est relativement humide et par conséquent les variations du taux d'infestation peuvent être plus importantes que dans la zone de Louga, située en climat sahélien, où le niveau d'infestation est vraisemblablement plus constant au cours de l'année. Globalement, les répétabilités étaient concordantes avec celles trouvées dans la bibliographie. Celles-ci varient entre 0,20 et 0,40 pour le nombre d'Opg (19) et entre 0,05 et 0,40 pour l'hématocrite (5). Elles peuvent parfois être beaucoup plus faibles, comme le montrent les résultats de Baker et coll. (6) selon lesquels les répétabilités sont de 0,22 pour l'hématocrite et 0,01 pour le nombre d'Opg. Sur les données du Sénégal, les critères analysés présentaient une répétabilité faible d'une année sur 
Tableau VIII

Estimation des corrélations phénotypiques (modèles à effets fixés) entre le nombre d'Opg et l'hématocrite pour différents prélèvements chez les mères et chez les jeunes

\begin{tabular}{|c|c|c|c|c|c|c|c|c|}
\hline \multirow[t]{2}{*}{ Echantillon } & \multicolumn{2}{|c|}{ Louga caprins } & \multicolumn{2}{|c|}{ Louga ovins } & \multicolumn{2}{|c|}{ Kolda Caprins } & \multicolumn{2}{|c|}{ Kolda ovins } \\
\hline & 1992 & 1993 & 1992 & 1993 & 1993 & 1994 & 1993 & 1994 \\
\hline \multicolumn{9}{|l|}{ Mères } \\
\hline $\begin{array}{l}\text { P1 } \\
\text { P3 } \\
\text { P1 + P3 }\end{array}$ & $\begin{array}{r}-0,08 \\
-0,30 \\
-\end{array}$ & $\begin{array}{c}-0,33 \\
0 \\
4\end{array}$ & $\begin{array}{r}-0,08 \\
-0,13 \\
-\end{array}$ & $\begin{array}{l}-0,20 \\
-0,08 \\
6\end{array}$ & $\begin{array}{l}-0,01 \\
-0,26\end{array}$ & $\begin{array}{l}-0,21 \\
-0,02 \\
2\end{array}$ & $\begin{array}{r}-0,17 \\
-0,21 \\
-\end{array}$ & $\begin{array}{l}-0,01 \\
0,03 \\
4\end{array}$ \\
\hline \multicolumn{9}{|l|}{ Jeunes } \\
\hline $\begin{array}{l}\mathrm{P} 1 \\
\mathrm{P} 3 \\
\mathrm{P} 1+\mathrm{P} 3\end{array}$ & $\begin{array}{r}-0,47 \\
-0,17 \\
-\end{array}$ & $\begin{array}{c}-0,12 \\
0,17\end{array}$ & $\begin{array}{r}-0,29 \\
-0,46 \\
-\end{array}$ & $\begin{array}{c}-0,19 \\
0,12 \\
5\end{array}$ & $\begin{array}{r}-0,25 \\
0,21 \\
-\end{array}$ & $\begin{array}{l}-0,04 \\
-0,19 \\
13\end{array}$ & $\begin{array}{r}-0,27 \\
-0,12 \\
-\end{array}$ & $\begin{array}{l}-0,22 \\
-0,10 \\
1\end{array}$ \\
\hline
\end{tabular}

Tableau IX

Estimation des corrélations phénotypiques (modèles à effets fixés) entre le poids et les deux autres caractères (nombre d'O pg et hématocrite) pour différents prélèvements chez les jeunes

\begin{tabular}{|c|c|c|c|c|c|c|c|c|}
\hline \multirow[t]{2}{*}{ Echantillon } & \multicolumn{2}{|c|}{ Louga caprins } & \multicolumn{2}{|c|}{ Louga ovins } & \multicolumn{2}{|c|}{ Kolda Caprins } & \multicolumn{2}{|c|}{ Kolda ovins } \\
\hline & 1992 & 1993 & 1992 & 1993 & 1993 & 1994 & 1993 & 1994 \\
\hline \multicolumn{9}{|c|}{ Poids - Nombre d'O pg } \\
\hline $\begin{array}{l}P 1 \\
P 2 \\
P 3 \\
P 1+P 2+P 3\end{array}$ & $\begin{array}{r}-0,20 \\
0 \\
-0,04 \\
-\end{array}$ & $\begin{array}{r}0,03 \\
0,25 \\
0,14 \\
8\end{array}$ & $\begin{array}{r}0,02 \\
-0,24 \\
-0,20 \\
-\end{array}$ & $\begin{array}{l}-0,03 \\
-0,17 \\
0,03 \\
2^{0}\end{array}$ & $\begin{array}{r}-0,19 \\
-0,02 \\
0,04\end{array}$ & $\begin{array}{l}-0,05 \\
-0,21 \\
0,79 \\
01\end{array}$ & $\begin{array}{r}-0,28 \\
-0,32 \\
0,04 \\
-\end{array}$ & $\begin{array}{l}-0,31 \\
-0,33 \\
-0,28 \\
5\end{array}$ \\
\hline \multicolumn{9}{|c|}{ Poids - Hématocrite } \\
\hline $\begin{array}{l}\text { P1 } \\
\text { P3 } \\
\text { P1 + P3 }\end{array}$ & $\begin{array}{l}0,31 \\
0,16\end{array}$ & $\begin{array}{l}0,07 \\
0,59\end{array}$ & $\begin{array}{l}0,31 \\
0,50\end{array}$ & $\begin{array}{r}0,17 \\
0,33 \\
5\end{array}$ & $\begin{array}{l}0,04 \\
0,01\end{array}$ & $\begin{array}{r}0,10 \\
0,12 \\
7\end{array}$ & $\begin{array}{l}0,16 \\
0,14\end{array}$ & $\begin{array}{l}0,19 \\
0,21 \\
\end{array}$ \\
\hline
\end{tabular}

l'autre, mais les effectifs des mères présentes deux années successives étaient faibles. D'autre part, les variations climatiques entre deux années successives peuvent être importantes, ce qui se répercute sur le parasitisme.

\section{Estimation de l'héritabilité}

Les valeurs d'héritabilité pouvaient être très élevées lorsqu'elles étaient estimées à partir d'un seul prélèvement, elles étaient proches de zéro lorsque les analyses étaient réalisées sur des prélèvements différents chez la mère et le jeune. D'autre part, les modèles incluant les effets aléatoires Env-com ou troup*P ont montré qu'il existait un effet d'environnement commun pour un prélèvement donné entre les individus sur le pâturage. D'après ces résultats, il semble exister des effets d'origine non génétique qui viennent soit augmenter, soit masquer la variabilité génétique. Dans le cas de l'analyse de mesures effectuées au même moment (P1, P2 ou P3) avec un modèle à effets fixés et un effet animal (modèle 1), la ressemblance entre individus pour les critères de résistance était à la fois d'origine génétique (relation mère-jeune) et d'origine environnementale (zones de pâturages communes au couple mère-jeune ou aux individus d'un même troupeau). Dans le cas de mesures réalisées à des moments différents, les covariances entre performances des mères et des jeunes incluaient des effets troupeau*saison inconnus et a priori beaucoup plus importants que les différences entre animaux. Dans le cas de mesures réalisées à plusieurs périodes $(\mathrm{P} 1+\mathrm{P} 2+\mathrm{P} 3)$ avec un modèle à effets fixés et un effet animal (modèle 1), la même pondération a été attribuée à des mesures effectuées au même moment (M1J1, M2J2, M3J3) et à des mesures effectuées à des moments différents (M1J2, M2J1, M1J3... ), alors que dans le premier cas il existait une covariation environnementale supplémentaire entre mères et jeunes. Sur l'échantillon P1 + P2 + P3, la prise en compte d'un effet d'environnement commun (Env-com du modèle 2 ou troup*P du modèle 3) a permis d'éliminer la variabilité d'origine environnementale pour un prélèvement donné, mais a eu aussi pour conséquence de retrancher de la variabilité génétique pour un prélèvement donné. D'une manière générale, pour une estimation de paramètres génétiques basée sur la covariance entre performances de la mère et des descendants, la non-prise en compte de l'effet troupeau au moment de la mesure s'est traduite par une surestimation de la ressemblance pour des couples de mesures effectuées au même moment : il devait exister une certaine similitude entre les environnements simultanés des deux groupes d'animaux. L'héritabilité était alors 
surestimée car la variance génétique estimée incluait une part de variance environnementale. A l'inverse, lorsqu'un effet d'environnement commun était pris en compte, cet effet englobait une partie de la variance génétique et l'héritabilité était sous-estimée.

La très petite taille des troupeaux interdisait la prise en compte, sous forme d'effet fixé, de leur impact sur les performances, car il n'était pas possible de distinguer correctement l'effet du milieu de l'effet des quelques individus composant le troupeau. Il n'était donc pas possible de considérer d'entrée cet effet troupeau en tant qu'effet fixé, ni même en tant qu'effet aléatoire, puisque dans cette dernière situation la majorité de la variance génétique aurait été éliminée.

Compte tenu des résultats, il est alors difficile de savoir lequel des deux effets (Env-com ou troup*P) reflète le mieux la situation. Dans le cas de petits troupeaux (avec deux ou trois couples mèrejeune), il y a peu de différence entre les effets troup*P et Env-com. Il n'y a pas d'exemple dans la bibliographie d'un tel effet d'environnement commun. L'étude de Bishop et coll. (8) s'en approche, puisqu'un effet maternel entre les descendants d'une même portée est inclus dans les modèles d'analyse. Cet effet intervient de façon importante dans la variation du nombre d'œufs excrétés et il tend à diminuer au fur et à mesure que les jeunes grandissent. L'influence de la mère agirait à plusieurs niveaux (8). Tout d'abord, la richesse du colostrum maternel fourni les premiers jours après la naissance influence non seulement la capacité du jeune à résister pendant les premiers mois de sa vie, mais aussi le développement de mécanismes de résistance. Ensuite, le choix des zones de pâturage par la mère et son comportement agissent sur la charge parasitaire à laquelle le jeune va être exposé. Enfin, de la production laitière de la mère va dépendre l'état général du jeune et ainsi sa capacité à se défendre contre une infestation. Pour l'étude des données du Sénégal, l'absence des paternités rendait impossible la prise en compte d'un effet maternel. Cependant, il aurait été utile de disposer d'informations supplémentaires sur la répartition spatiale des animaux au pâturage, pour savoir notamment si les animaux d'un troupeau restent groupés entre eux lorsqu'ils sont laissés en divagation pendant la journée ou si le couple mère-jeune est prédominant.

Les héritabilités estimées dans ce travail étaient pour certaines du même ordre de grandeur que celles obtenus dans d'autres études. Par exemple, Baker et coll. (7) et Baker (4), obtiennent des héritabilités comprises entre 0,01 et 0,35 chez différentes races ovines kenyanes et éthiopiennes. Il n'a pas été possible de mettre en évidence des différences entre les paramètres génétiques des caprins et des ovins, la variabilité des résultats étant trop importante.

Dans toutes les situations étudiées dans ce travail, l'héritabilité a été sous-estimée. En effet, la variance de l'effet aléatoire troup*P ou la variance de l'effet Env-com entre mère et jeune ont été prises en compte, lors du calcul de l'héritabilité, au niveau de la variance phénotypique (dénominateur) mais pas au niveau de la variance génétique (numérateur), alors que ces effets incluent une part de variabilité d'origine génétique. D'autre part, en raison de la valeur 0,60 donnée par Bouix et coll. (11) et Morris et coll. (24) pour la corrélation génétique entre résistance des mères et des jeunes, les estimations d'héritabilité trouvées étaient une estimation minorée (vraisemblablement de 40 p. 100) des vraies valeurs.

\section{Estimation des corrélations phénotypiques}

Le nombre d'Opg et l'hématocrite étaient négativement corrélés, avec des valeurs moyennes de l'ordre de - 0,11 chez les jeunes et - 0,21 chez les mères. Ce résultat concorde avec le fait que le parasite hématophage Haemonchus contortus est prédominant sur le pâturage. Chez de jeunes chevreaux Créole de Guadeloupe, Man- donnet et coll. (23) ont estimé des corrélations phénotypiques de l'ordre de - 0,29 entre les mêmes caractères de résistance pour une infestation par Haemonchus contortus et Trichostrongylus colubriformis.

Le poids, bien que corrigé pour les effet fixés du modèle (troupeau*année*saison, âge, sexe et mode de naissance), était négativement corrélé avec le nombre d'Opg (- 0,14 en moyenne) et positivement avec l'hématocrite ( 0,23 en moyenne). Ces résultats indiquent que le parasitisme intervient comme un facteur de réduction de la croissance, ou inversement que les animaux les plus lourds, et donc les mieux nourris, résistent mieux à l'infestation. Ces estimations sont concordantes avec celles de Mandonnet et coll. (23) qui estiment des corrélations phénotypiques de - 0,09 et - 0,06 entre le poids et le nombre d'œufs excrétés, et une corrélation de 0,36 entre le poids et l'hématocrite.

\section{Fiabilité des critères de résistance}

Le critère nombre d'Opg n'était pas toujours bien corrélé avec la charge parasitaire. La méthode de conservation ainsi que la répartition des œufs dans les voies digestives peuvent être des sources d'erreur au moment du dénombrement. Aumont et coll. (2) ont montré que la conservation des fèces dans une solution de formaldéhyde pouvait entraîner une sous-estimation du nombre d'œufs de parasites, les animaux peu infestés ne pouvant alors être dissociés des animaux non infestés. La répartition de la ponte dans les voies digestives n'est pas toujours homogène et si les fèces sont prélevées en quantité insuffisante, le nombre d'œufs dénombrés ne sera pas révélateur du niveau d'infestation de l'animal. Enfin, la troisième source d'imprécision du nombre d'Opg peut intervenir après une première infestation lors d'un self-cure. Ce phénomène est caractérisé par une expulsion massive de parasites, le nombre d'Opg étant élevé alors que la charge parasitaire est faible (1) Malgré tout, le caractère nombre d'Opg garde une certaine validité si l'on considère la constance des valeurs d'héritabilité et de répétabilité publiées. En tout état de cause, ce caractère est une mesure directe de la contamination des pâturages par les animaux.

Le critère hématocrite peut avoir d'autres sources de variation que le parasitisme. Des facteurs saisonniers (alimentation, état physiologique, etc. ) ou la présence de maladies telles que la trypanosomose peuvent agir sur le taux d'hématocrite et ainsi se confondre avec l'infestation par des parasites hématophages.

\section{- CON CLU SION}

Ce travail a mis en évidence un certain nombre de difficultés relatives à la mesure de la variabilité génétique de la résistance au parasitisme gastro-intestinal chez des petits ruminants en milieu difficile. Le partitionnement des données en petites unités, comme c'était le cas ici pour les troupeaux, est un obstacle majeur à une estimation correcte des paramètres génétiques. Avec une telle structure de données, la prise en compte d'un effet troupeau ne permet pas de dissocier de façon satisfaisante la variabilité environnementale créée par l'effet du troupeau et la variabilité génétique. Les estimations auraient été plus fiables si le recueil des données avait été réalisé sur des troupeaux de plus grande taille (pour un même nombre d'animaux examinés), ou à défaut, si des informations complémentaires sur les pratiques d'élevage et sur le comportement des animaux au pâturage avaient été disponibles. La connaissance du mode de conduite - divagation et/ou conduite sur pâturage en troupeaux villageois - et de la répartition spatiale des animaux - notamment du couple mère-jeune - aurait permis de redéfinir une unité d'élevage plus cohérente et homogène que le regroupement des troupeaux d'un même village. Par ailleurs, le 
recueil de données chez des femelles et chez leurs descendants au même âge aurait permis de s'affranchir du problème de l'inégalité des variances pour l'hématocrite et de lever les doutes sur la différence entre les caractères de résistance des jeunes et des adultes, mais cette approche est plus délicate à envisager dans un tel milieu.

Les résultats obtenus dans cette étude indiquent qu'il existe une variabilité individuelle importante et qu'il semble exister une variabilité génétique de la résistance des petits ruminants aux parasites gastro-intestinaux chez les races sénégalaises, même si elle est difficile à estimer dans de telles conditions. Si une action de sélection devait être envisagée, il serait nécessaire d'approfondir ces résultats et de s'intéresser à des unités d'élevage plus grandes pour réaliser une évaluation génétique. La sélection pour la résistance au parasitisme gastro-intestinal dans les pays tropicaux permettrait d'intégrer dans les schémas d'amélioration génétique des races rustiques les qualités d'adaptation des animaux qui constituent un facteur essentiel en milieux difficiles (10).

\section{BIBLIO GRAPHIE}

1. ALLONBY E.W., URQUHART G.M., 1975. The epidemiology of

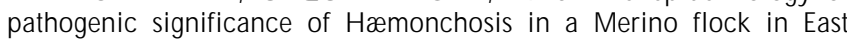
Africa. Vet. Parasitol., 1: 129-143.

2. AUMONT G., BARRE N., DUAW S., OUATHARA L., POUILLOT R., TILLARD E., VASSILIADES G., 1995. Preservation of faecal samples and laboratory sources of variation in faecal strongyles egg counts of small ruminants from tropical regions. In: International Conference, Nove approaches to the control of Helminth parasites of livestock, UNECSIRO, Armidale, Australie, 18-21 April 1995, p. 19.

3. AUMONT G., MANDONNET N., MAHIEU M., VARO H., 1997. Le periparturient rise chez les caprins et les ovins de Guadeloupe et de Martinique (F.W.I.), résultats préliminaires. Réunion Atp Mipa, Strongyloses gastro-intestinales des petits ruminants en milieu tropical, Petit Bourg, Guadeloupe, 16-20 juin 1997.

4. BAKER R.L., 1999. A review of genetic resistance to gastrointestinal nematode parasites in sheep and goats in the tropics and evidence for resistance in some sheep and goat breeds in sub-humid coastal Kenya. Anim. G enet. Res. Inf., 24: 13-30.

5. BAKER R.L., LAHLOU KASSI A., REGE J.E.O., REYNOLDS L., BEKELE T., MUKASSA-MUGERWA E., REY B., 1992. A review of genetic resistance to endoparasites in small ruminants and an outline of ILCA's research programme in this area. In: Proc. 10th Scientific Workshop of the Small Ruminants Collaborative Research Support Programme, Nairobi, Kenya, 26-27 February 1992, p. 79-104.

6. BAKER R.L., MWAMACHI D.M., AUDHO J.O., ADUDA E.O., THORPE W., 1998. Genetic resistance to gastrointestinal nematode parasites in Red Maasai, Dorper and Red Maasai x Dorper ewes in the sub-humid tropics. Anim. Sci. (soumis).

7. BAKER R.L., REGE J.E.O., TEMBELY S., MUKASA-MUGERWA E., ANINDO D., MWAMACHI D.M., THORPE W., LAHLOU-KASSI A., 1998. Genetic resistance to gastrointestinal nematode parasites in some indigenous breeds of sheep and goats in East Africa. Proc. 6th WId Congr. Genet. Appl. Livest. Prod., XXV: 269-272.

8. BISHOP S.C., BAIRDEN K., MCKELLAR Q.A., PARK M., STEAR M.J., 1996. Genetic parameters for faecal egg count following mixed, natural, predominantly 0 stertagia circumcincta infection in relationships with live weight in young lambs. Anim. Sci., 63: 423-428.

9. BISSET S.A., MORRIS C.A., 1996. Feasibility and implications of breeding sheep for resilience to nematode challenge. Int. J. Parasitol., 26 (8-9): 857-868.

10. BOUIX J., 1992. Adaptation des ovins aux conditions de milieu difficiles. Inra Prod. Anim., hors série : Eléments de génétique quantitative et applications aux populations animales, p. 179-184.

11. BOUIX J., KRUPINSKI J., RZEPECKI R., NOWOSAD B., SKRZYZALA I., ROBORZYNSKI M., FUDALEWICZ-NIEMCZYK W., SKALSKA M., MALCZEWSKI A., GRUNER L., 1998. Genetic resistance to gastrointestinal nematode parasites in a polish long-wool sheep. Int. J. Parasitol., 28: 1797-1804.
12. CHARTIER C., BUSHU M., LUBINGO M., 1990. Principaux helminthes des petits ruminants en Ituri (Haut-Zaïre). Ann. Soc. Belge M éd. Trop., $70: 65-75$.

13. COURTNEY C.H., GESSNER R., SHOLTZ S.R., LO GGINS P.E., 1986. The periperturient rise in faecal egg counts in three strains of Florida native ewes and its value in predicting resistance of lambs to Haemonchus contortus. Int. J. Parasitol., 16 (3): 185-189.

14. DE VANEY J.A., CRAIG T.M., ROWE L.D., 1992. Resistance to ivermectine by Haemonchus contortus in goats and calves. Int. J. Parasitol., 22 (3): 369-376.

15. FAUGERE 0., DOCKES A.C., PERROT C., FAU GERE B., 1990a. L'élevage traditionnel des petits ruminants au Sénégal. I. Pratiques de conduite et d'exploitation des animaux chez les éleveurs de la région de Kolda. Rev. Elev. Méd. vét. Pays trop., 43 : 249-259.

16. FAU GERE O., DOCKES A.C., PERROT C., FAU GERE B., 1990b. L'élevage traditionnel des petits ruminants au Sénégal. II. Pratiques de conduite et d'exploitation des animaux chez les éleveurs de la région de Louga. Rev. Elev. Méd. vét. Pays trop., 43 : 261-273.

17. GRUNER L., 1991. Breeding for helminth resistance in sheep and goats. In: 0 wen J.B., Axford R.F.E., eds. Breeding for Disease Resistance in Farm Animals, Oxford, UK, CAB International, p. 187-200.

18. GRUNER L., BOUIX J., CABARET J., BOULARD C., CORTET J., SAUVE C., MOLENAT G., CALAMEL M., 1992. Effect of genetic type, lactation and management on helminth infection of ewes in an intensive grazing system on irrigated pasture. Int. J. Parasitol., 22 (7): 919-925.

19. GRUNER L., BOUIX J., VU TIEN KHANG J., 1997. La résistance génétique aux parasitoses internes : exemples de travaux engagés en France et en Pologne. Point Vét., 28 : 1-7. ( ${ }^{\circ}$ spécial)

20. GRUNER L., MANDONNET N., BOUIX J., VU TIEN KHANG J., CABARET J., HOSTE H., KERBOEUF D., BARNOUIN J., 1994. W orm population characteristics and pathological changes in lambs after a single or trickle infection with Teladorsagia circumcincta. Int. J. Parasitol., 24 (3): 347-356.

21. MAINGI N., 1991. Resistance to thiabendazole, fenbendazole and levamisole in Haemonchus contortus and Trichostrongylus species in sheep on a Kenyan farm. Vet. Parasitol., 39: 285-291.

22. MANDONNET N., 1995. Analyse de la variabilité génétique de la résistance aux strongles gastro-intestinaux chez les petits ruminants. Eléments pour la définition d'objectifs et de critère de sélection en milieu tempéré ou tropical. Thèse Doct. Sci., U niversité Paris-Sud, France, 115 p.

23. MANDONNET N., AUMONT G., FLEURY J., GRUNER L., BOUIX J., VU TIEN KHANG J., 1996. Genetic variability in resistance of Creole goats to natural infection with Trichostrongylids in Guadeloupe. In: Camus E., House J.A., Uilenberg G. (eds). Vector-borne pathogens: international trade and tropical animal disease, New-York, USA, The New-York Academy of Science, p. 421-431.

24. MORRIS C.A., BISSET S.A., VLASSO FF A., WEST C.J., WHEELER M., 1998. Faecal nematode egg counts in lacting ewes from Romney flocks selectively bred for divergence in lamb faecal egg count. Anim. Sci., 67: 283-288.

25. MOU LIN C.H., 1993. Performances animales et pratiques d'élevage en Afrique sahélienne. La diversité du fonctionnement des troupeaux de petits ruminants dans la communauté rurale de $\mathrm{N}$ diagne (Sénégal). Thèse Doct. Sci., Ina Paris-Grignon, France, 259 p.

26. MOULIN C.H., FAUGERE O., FAUGERE B., 1994. L'élevage traditionnel des petits ruminants au Sénégal. III. Pratiques de conduite et d'exploitation des animaux chez les éleveurs de la communauté rurale de Kaymor (Siné-Saloum, Sénégal). Rev. Elev. Méd. vét. Pays trop., 47 : 223-234.

27. NDAMUKONG K.J.N., SEWELL M.M.H., 1992. Resistance to benzimidazole anthelmintics by trichostrongyles in sheep and goats in N orth-W est Cameroon. Vet. Parasitol., 41: 335-339.

28. NEUM AIER A., GRO EN VELD E., 1998. Restricted maximum likelihood of covariances in sparse linear model. Genet. Sel. Evol, 30: 3-26.

29. ROMIALI E., PANDEY V.S., GATENBY R.M., DOLOKSARIBU M., SAKUL H., WILSON A., VERHULST A. 1997. Genetic resistance of different genotypes of sheep to natural infections with gastrointestinal nematodes. Anim. Sci., 64: 97-104.

30. STATISTICAL ANALYSIS SYSTEM, 1990. SAS U ser's guide: statistics. Cary, NC, USA, SAS Institute, $1028 \mathrm{p}$. 
31. TILLARD E., 1991. Prophylaxies chez les petits ruminants au Sénégal : évaluation technico-économique de leurs effets en milieu villageois. lemvt/Inra/Enva/M nhn, $1: 212$.

32. WO OLASTON R.R., 1992. Selection of Merino sheep for increased and decreased resistance to Haemonchus contortus: peri-parturient effects on faecal egg counts. Int. J. Parasitol., 22 (7): 947-953.

\section{Summary}

Clément V., Bouix J., Tillard E., Gueye A., Baker R.L. Genetic parameters estimation of resistance to gastrointestinal parasitism in traditional husbandry system of small ruminants in Senegal

A protocol has been carried out in order to study the genetic resistance to gastrointestinal parasites on Senegalese breeds of sheep and goats. Faecal and blood samples have been collected on animals from different flocks of two areas of Senegal: the area of Louga located in a sahelian climate, and the area of Kolda located in a sudano-guinean climate. Sires identity was unknown in the husbandry system and pedigree were primarily based on dam-offspring relation. Traits analysed were faecal egg count (FEC) and packed cell volume (PCV). The study was conducted during two years: 1992-1993 for animals of Louga and 1993-1994 for animals of Kolda, with three samplings each year for FEC, two for PCV during rainy season. In Louga area, 193 goats and 184 sheep were collected the first year, 249 goats and 357 sheep were collected the second year, which corresponded to 60 flocks distributed in 15 villages. In Kolda area, the number of animals sampled was 322 goats and 349 sheep the first year, 297 goats and 428 sheep the second year. These animals were coming from 80 flocks distributed in 25 villages. Factors of variation of performances have been fitted in analysis models: village*season*year, age and lactation level for dams, village* season*year, age and sex for young. The heritability and the repeatability have been estimated using an "animal model". The mean of the repeatability within year is 0.19 for FEC and 0.27 for PCV. Values of repeatability were smaller between years: 0.12 for FEC and 0.21 for PCV. The estimation of heritability was difficult: data structure didn't allow to fit directly maternal environment and flocks effects, because of insufficient pedigree and small size of the flocks, respectively. Values of heritability estimated varied from 0 to 0.33 for FEC and from 0 to 0.38 for PCV, depending on sample analysed and model used. It was not possible to estimate genetic correlation between traits. However phenotypic correlations have been estimated between FEC and PCV, and between each one of these variables and the liveweight of the young. Constraints related to genetic study in traditional husbandry system have been discussed.

Key words: Sheep - Goat - Animal husbandry methods Parasites - Digestive system diseases - Genetic parameter Senegal.
33. YADAV C.L., UPPAL R.P., KALRA S., 1993. An outbreak of Haemonchosis associated with anthelmintic resistance in sheep. Int. J. Parasitol., 23 (3): 411-413.

Reçu le 7-6-1999, accepté le 16-11-99

\section{Resumen}

Clément V., Bouix J., Tillard E., Gueye A., Baker R.L. Estimación de los parámetros genéticos de la resistencia al parasitismo gastrointestinal en un medio de crianza aldeano de pequeños rumiantes en Senegal.

Se estableció un protocolo, con el objetivo de estudiar la resistencia genética a las estrongilosis gastrointestinales de las razas locales de ovinos y caprinos de Senegal. Se llevaron a cabo tomas de sangre y coproscopias, en animales pertenecientes a diferentes hatos, en dos regiones de Senegal: Ia zona de Louga, con clima sahelino y la zona de Kolda, con clima sudano guineense. En este contexto de crianza, la identidad de los padres fue conocida, las relaciones de parentesco estudiadas fueron esencialmente la de madre-descendiente. Los caracteres estudiados fueron la coproscopia en número de huevos por gramo de heces y el hematocrito. La duración del estudio fue de dos años: 1992-1993 para los animales de Louga y 1993-1994 para los animales de Kolda, a razón de tres muestras por año para la coproscopia y de dos para el hematocrito, repartidas durante la estación de Iluvias. En la región de Louga, el número de individuos de los que se obtuvo una muestra fue de 193 para los caprinos y de 184 para los ovinos durante el primer año, 249 para los caprinos y 357 para los ovinos durante el segundo año, lo que corresponde a una muestra de 60 establecimientos repartidos en 15 aldeas. En la zona de Kolda, el estudio concernió 322 caprinos y 349 ovinos durante el primer año, 297 caprinos y 428 ovinos durante el segundo año. Estos animales provenían de 80 hatos repartidos en 25 aldeas. Los factores de variación de los rendimientos tomados en cuenta en los modelos de análisis fueron: la combinación aldea*estación* año, la edad y el estadio de lactación de las madres, la combinación aldea*estación*año, la edad y el sexo para los jóvenes. La heredabilidad y la repetibilidad se estimaron gracias a un modelo "animal". La repetibilidad media intra-año fue de 0,19 para el carácter número de huevos por gramo de heces y de 0,27 para el hematocrito. Esta fue más baja entre años, del orden de 0,12 para el número de huevos por gramos de heces y de 0,21 para el hematocrito. La estimación de la heredabilidad presentó dificultades, debido a la estructura de los datos aldeanos, los cuales no permitían una estimación directa el medio materno y de los efectos de hato, a causa de genealogías insuficientes y a un número bajo de efectivos por establecimiento, respectivamente. Las estimaciones de la heredabilidad variaron entre 0 y 0,33 para el número de huevos por gramo de heces y entre 0 y 0,38 para el hematocrito, según la muestra analizada y el modelo utilizado. No fue posible obtener correlaciones genéticas entre los caracteres. En revancha, las correlaciones fenotípicas fueron estimadas entre el número de huevos por gramo de heces y el hematocrito, así como entre cada una de estas dos variables y el peso de los jóvenes. Se discuten los obstáculos ligados a un estudio genético en medio tradicional.

Palabras clave: O vino - Caprino - M etodos de crianza - Parasitos - Enfermedades del systema del sistemo digestivo - Parámetro genético - Senegal. 